\title{
Editorial
}

Nephrology

Published online: October 22, 2016

DOI: $10.1159 / 000450863$

\section{Magnesium and Kidney Health - More on the 'Forgotten Electrolyte'}

\author{
Jürgen Vormann \\ Institute for Prevention and Nutrition, Ismaning/Munich, Germany
}

Magnesium is the fourth most abundant cation and is essential for every cell [1]. This mineral is a cofactor in multiple enzymatic reactions, including those involving energy metabolism as well as DNA and protein synthesis, and it participates in the regulation of ion channels. Magnesium homeostasis is therefore fundamental to the existence of life. However, the clinical significance of magnesium has only been acknowledged during recent years. In fact, years ago, magnesium was even called 'the forgotten electrolyte' [2]. In this issue of the American Journal of Nephrology, Rebholz et al. [3] report on the correlation between dietary magnesium intake and kidney function decline from a large epidemiological investigation, the HANDLS study. Compared with the upper tertile of dietary magnesium intake, the risk for rapid kidney function decline was roughly doubled in the lower tertile. This result adds to a growing number of epidemiological studies connecting increased risk for various diseases with a low dietary magnesium intake or low serum magnesium concentration. Up to now, these correlations were reported for diabetes type 2, stroke, cardiovascular diseases and even sudden cardiac death (reviewed in [4]). The relevance of these findings is important as the general magnesium intake in the western population is low. Dietary data suggest that the average magnesium intake has declined markedly over the last 100 years. Studies in the US showed that $23.5 \%$ of the population had a daily dietary magnesium intake of less than $50 \%$ of the recommendations. Similar trends to low dietary magnesium intake were found in UK and Germany. The daily magnesium intakes below recommendations increased from $25 \%$ of the total population to about $50 \%$.

\section{KARGER}

E-Mail karger@karger.com

www.karger.com/ajn
Whereas in the general population magnesium deficits are most often thought to result mainly in calf cramps (which are generally not considered to be life threatening), it is less well known that a magnesium deficit induced by a magnesium-deficient diet in humans resulted in severe cardiac arrhythmias [5]. Other studies as, for example, an epidemiological study in Germany showed that low serum magnesium levels were associated with higher all-cause and, especially, cardiovascular mortality [6]. A sufficiently high magnesium intake and magnesium status, therefore, seems to be lifesaving.

The connection between magnesium and the kidney is manifold. More than being regulated by intestinal absorption, magnesium homeostasis is governed by reabsorption of magnesium from primary urine in the kidney. Of about $2,400 \mathrm{mg}$ magnesium that is being ultra-filtrated daily, 95-99\% must be reabsorbed by the nephrons. The mechanisms responsible for the fine-tuning have been elucidated during recent years and primarily involve transport systems located in the thick ascending limb of Henle's loop and distal convoluted tubules [4]. If the magnesium intake is low, primarily the kidneys avoid rapid and dramatic falls in extracellular magnesium concentrations by increasing the amount of magnesium reabsorption. Therefore, with low magnesium intakes the magnesium concentration in urine is significantly reduced. This is achieved by increased expression and production of magnesium transporters.

Editorial based on: Rebholz CM, Tin A, Liu Y, Fanelli-Kuczmarski MT, Evans MK, Zonderman AB, Crews DC: Dietary magnesium and kidney function decline: the HANDLS study.

Prof. Dr. Jürgen Vormann

Institute for Prevention and Nutrition Adalperostr. 37

DE-85737 Ismaning/Munich (Germany)

E-Mail vormann@ipev.de 
Renal magnesium wasting caused by genetic defects in these transport systems were classified as hypercalciuric hypomagnesemia, Gitelman-like hypomagnesemia, mitochondrial hypomagnesemia and other hypomagnesemia [7]. In addition, it is now also known that several drugs like diuretics, proton pump inhibitors, EGF-receptor antagonists, cyclosporine, cisplatin and several antibiotics induce severe magnesium losses by various mechanisms.

The evidence of a well-functioning kidney for proper magnesium homeostasis is obvious and a decline in kidney function might also significantly influence magnesium homeostasis. On the other hand, how a low magnesium status affects kidney health is less well known. The possible mechanisms are numerous as magnesium affects nearly all biochemical pathways. Rebholz et al. [3] discuss effects of hypomagnesemia concerning blood vessels and epithelia. From animal experiments, it is well known that feeding a severely magnesium-deficient diet will induce a pro-inflammatory and pro-oxidative state leading to tissue injury. It is also known from these experiments that magnesium deficiency induces calcification of the kidney. In humans with low magnesium status, markers of inflammation - like C-reactive protein - are increased. An increased secretion of catecholamines is also observed due to the reduced calcium antagonism of magnesium, resulting in constriction of renal blood vessels.

In their investigations, Rebholz et al. [3] could not measure serum magnesium concentrations. It would be interesting to know whether the same correlation as with dietary magnesium intake would also be observed with serum magnesium concentration. However, serum magnesium represents only $1 \%$ of total magnesium and poorly reflects intracellular magnesium concentrations. Whether intracellular magnesium deficits could also contribute to the loss of kidney function remain to be investigated. Reduced magnesium levels in the blood can lead to an increased expression of magnesium transporters in the kidney. By this mechanism, low serum magnesium concentrations can be avoided at least for a while. It could be speculated that a long-term overexpression of these magnesium transporters could lead to changes in cellular homeostasis, finally leading to damage of nephrons. The calcium antagonistic effect of magnesium is also important for reducing the risk of kidney stones, and silent kidney stones significantly increase the risk of kidney failure. As low magnesium intake will reduce the urinary magnesium concentration, the beneficial effect of magnesium on stone formation is hampered. This effect will already be working when the serum magnesium concentration is still close to normal. So it is reasonable to assume that several mechanisms will add together in sensitive subjects with low dietary magnesium intake - at the end resulting in irreversible kidney damage.

Generally, from a public health point of view, it is disturbing that the usual dietary intake of magnesium is very low, especially in the context of a usually relatively high intake of calcium. It might be worthwhile to try to reduce the calcium/magnesium ratio in the diet by increasing the magnesium intake. Probably, just by this a significant protective effect could be achieved. It would also be important to investigate if the incidence of kidney failure would be reduced in populations using magnesium supplements compared to non-users. Prospective intervention studies with magnesium supplementation could indicate the contribution of this mineral to disease risks. Intervention studies concerning prevention of disease, however, will be unlikely to be performed because of the need for long duration of a placebo-controlled setting.

Studies like that of Rebholz et al. [3] significantly increase the knowledge on the importance of the formerly 'forgotten electrolyte'. Even if there is no definite answer on mechanisms and singular effects of magnesium on kidney health, these data should increase the awareness about the possible beneficial effects of a high dietary magnesium intake. Furthermore, there should be a closer look on magnesium in studies on the pathophysiological mechanisms resulting in kidney damage.

\section{References}

1 Vormann J: Magnesium: nutrition and homoeostasis. AIMS Public Health 2016;3:329340.

2 Elin RJ: Magnesium: the fifth but forgotten electrolyte. Am J Clin Pathol 1994;102:616622.

3 Rebholz CM, Tin A, Liu Y, Fanelli-Kuczmarski MT, Evans MK, Zonderman AB, Crews DC: Dietary magnesium and kidney function decline: the HANDLS study. Am J Nephrol 2016;44:381-387.
4 de Baaij JH, Hoenderop JG, Bindels RJ: Magnesium in man: implications for health and disease. Physiol Rev 2015;95:1-46.

5 Nielsen FH, Milne DB, Klevay LM, Gallagher S, Johnson L: Dietary magnesium deficiency induces heart rhythm changes, impairs glucose tolerance, and decreases serum cholesterol in post menopausal women. J Am Coll Nutr 2007;26:121-132.
6 Reffelmann T, Ittermann T, Dörr M, Völzke H, Reinthaler M, Petersmann A, Felix SB: Low serum magnesium concentrations predict cardiovascular and all-cause mortality. Atherosclerosis 2011;219:280-284.

7 Viering DH, de Baaij JH, Walsh SB, Kleta R, Bockenhauer D: Genetic causes of hypomagnesemia, a clinical overview. Pediatr Nephrol 2016, Epub ahead of print. 